\title{
Correlation of cytomorphology and histopathology in the diagnostic process of myeloid malignancies
}

\author{
Laura Engelbrecht ${ }^{1,2}$, Katharina S Götze ${ }^{3}$, Kristina Schwamborn ${ }^{4}$, Catharina Müller-Thomas ${ }^{3}$, Tamara Alpermann ${ }^{1}$, Nicolas Bonadies ${ }^{1,5}$ \\ Myriam Legros ${ }^{1}$, Johanna Flach', Thomas Pabst ${ }^{7}$, Yara Banz ${ }^{2, a}$ and Ulrike Bacher ${ }^{1, b *}$ \\ ${ }^{1}$ Department of Hematology and Central Hematology Laboratory, Inselspital, Bern University Hospital, University of Bern, Bern, Switzerland \\ ${ }^{2}$ Institute of Pathology, University of Bern, Bern, Switzerland \\ ${ }^{3}$ Department of Medicine III, Klinikum Rechts der Isar, Technical University of Munich, Munich, Germany \\ ${ }^{4}$ Institute of Pathology, Klinikum Rechts der Isar, Technical University of Munich, Munich, Germany \\ ${ }^{5}$ Department for BioMedical Research, University of Bern, Bern, Switzerland \\ ${ }^{6}$ Department of Hematology and Oncology, Medical Faculty Mannheim of the Heidelberg University, Mannheim, Germany \\ ${ }^{7}$ Department of Medical Oncology, Inselspital, Bern University Hospital, University of Bern, Bern, Switzerland \\ a,bUB and YB contributed equally.
}

\begin{abstract}
Bone marrow cytomorphology and histopathology are the cornerstones for the initial diagnosis of myelodysplastic syndromes (MDS) and other related myeloid disorders. They provide a rapid first insight into diagnostic categories and thus help in clinical decision making. However, difficulties in the morphologic assessment of MDS exist due to inter- and intra-observer variability. In this study, we directly compared the results of cytomorphology and histopathology obtained in a realworld diagnostic scenario in 90 patients with myeloid malignancies aiming to evaluate their validity for diagnosing and classifying various myeloid malignancies. While both techniques placed $80 \%$ of our bone marrow samples into the same diagnostic category and thus showed a good correlation, our study also demonstrates the limitations in correlating marrow cytomorphology and histopathology, even following stringent and repetitive diagnostic assessments. This was particularly true for CMML, where not only additional diagnostic tools such as molecular genetics or clinical evaluation but also the analysis of the peripheral blood smears aided in finding the correct diagnosis. Overall, our data emphasize the need for a comprehensive diagnostic review in a patient-for-patient setting when a myeloid malignancy is suspected or confirmed. We propose that the combination of cytomorphologic and histopathologic assessment with clinical, laboratory, and genetic parameters is essential in achieving high diagnostic accuracy in an interdisciplinary setting.
\end{abstract}

\section{Introduction}

Myelodysplastic syndromes (MDS) represent clonal hematopoietic stem cell disorders with cytopenia, varying degrees of dysplasia, and frequent transformation towards secondary acute myeloid leukemia (s-AML) [1]. Cytomorphology, histopathology, and immunohistochemistry are the major initial diagnostic cornerstones for MDS [2] as well as related myeloid disorders such as myelodysplastic/ myeloproliferative neoplasm (MDS/MPN)-overlap conditions including chronic myelomonocytic leukemia (CMML). As interpreting cytomorphology and histopathology remains challenging in many cases, repeated follow-up investigations may be necessary to confirm MDS diagnosis. Considering the increasing frequency of MDS due to aging of the general population [3-6] rapid and efficient diagnostics gain importance for myeloid malignancies. Next-generation sequencing (NGS) was integrated into the contemporary diagnostic process [7-9], but comes with significant costs, requires an intensive post-analytical work-up, and should always be aligned with other diagnostic methods [10]. Furthermore, the phenomenon of clonal hematopoiesis of indeterminate potential $[11,12]$ emphasizes that somatic mutations per se do not necessarily confirm MDS diagnosis.

Difficulties in the morphologic diagnosis of MDS result from inter- and intra-observer variability in the determination of unilineage dysplasia [13,14], especially in the absence of ring sideroblasts or increased blasts. We here compared the results of cytomorphology and histopathology obtained in a real-world diagnostic scenario in 90 patients with MDS and the spectrum of related disorders aiming to evaluate their validity for diagnosing and classifying various myeloid malignancies.

\section{Materials and Methods}

We included 90 patients diagnosed with a myeloid malignancy at the university hospitals Bern and Munich in the period of 20072017. Requirement for inclusion in the retrospective study was the availability of both bone marrow cytomorphology and histopathology. The following categories were considered: all subtypes of MDS and MDS/MPN as well as CMML and s-AML (Tables 1 and 2). Patients

${ }^{\star}$ Correspondence to: Ulrike Bacher, MD, Associate Professor; Center of Laboratory Medicine (ZLM), Inselspital, Bern University Hospital, University of Bern; Bern, Switzerland, Tel: +41-31-632-1390; Fax. +41-31-632-3406; E-mail: veraulrike.bacher@insel.ch

Key words: Myelodysplastic syndromes (MDS), cytomorphology, histopathology, diagnostic algorithm, correlation

Received: September 29, 2019; Accepted: October 10, 2019; Published: October 14,2019 
Table 1. Demographic features, peripheral blood parameters, risk profiles, and genetic results in the study cohort

\begin{tabular}{|c|c|}
\hline Parameter & Number (\%) \\
\hline Patients & $90(100.0 \%)$ \\
\hline At diagnosis & $87(96.7)$ \\
\hline At follow-up & $3(3.3)$ \\
\hline Males/females (ratio) & $56 / 34(1.6)$ \\
\hline Age, years, median (range) & $70(21-87)$ \\
\hline \multicolumn{2}{|l|}{ Peripheral blood parameters } \\
\hline WBC, G/L, median (range) & $3.4(0.1-87)$ \\
\hline $\mathrm{Hb}, \mathrm{g} / \mathrm{L}$, median (range) & $91(58-153)$ \\
\hline Thrombocytes, g/l, median (range) & $57(1-958)$ \\
\hline IPSS risk group (in MDS pts) & $59(100.0 \%)$ \\
\hline Low & $18(30.5)$ \\
\hline intermediate-1 & $22(37.3)$ \\
\hline intermediate- 2 & $17(28.8)$ \\
\hline High & $2(3.4)$ \\
\hline IPSS-R risk group (in MDS pts) & $55(100.0 \%)$ \\
\hline Very poor & $13(23.6)$ \\
\hline Poor & $9(16.4)$ \\
\hline Intermediate & $15(27.3)$ \\
\hline Good & $17(30.9)$ \\
\hline Very good & $1(1.8)$ \\
\hline Cytogenetics & $85(100.0 \%)$ \\
\hline Normal karyotype & $30(35.3)$ \\
\hline Aberrant karyotypes & $55(64.7)$ \\
\hline$-\mathrm{Y}$ & $3(5.5)$ \\
\hline$-5 / \operatorname{del}(5 q)$ & $4(7.3)$ \\
\hline $\operatorname{del}(20 q)$ & $3(5.5)$ \\
\hline$-7 / \operatorname{del}(7 q)$ & $10(18.2)$ \\
\hline Complex $(\geq 3)$ aberrations & $17(30.9)$ \\
\hline Other aberrations & $18(32.7)$ \\
\hline
\end{tabular}

Del: deletion, $\mathrm{Hb}$ : Hemoglobin; IPSS: International Prognostic Scoring System; IPSS-R: Revised International Prognostic Scoring System; WBC: White blood cells.

Table 2. Classification of the disorders according to cytomorphology and histopathology

\begin{tabular}{|l|l|l|}
\hline \multicolumn{2}{|l|}{ Number (\%) } & Histopathology \\
\hline Parameter & Cytomorphology & N=34 (\%) \\
\hline Diagnostic category 1 & N=31 (\%) & $10(11.1)$ \\
\hline RCMD & $15(16.7)$ & - \\
RARS & $1(1.1)$ & - \\
RARS-T & $1(1.1)$ & $2(2.2)$ \\
Hypoplastic MDS & $2(2.2)$ & $1(1.1)$ \\
MDS with isolated 5q deletion & $1(1.1)$ & $1(1.1)$ \\
MDS/MPN overlap & $3(3.3)$ & $20(22.2)$ \\
\hline Suspected MDS & $8(8.9)$ & $\mathbf{N}=\mathbf{2 5}(\mathbf{\%})$ \\
\hline Diagnostic category $\mathbf{2}$ & $\mathbf{N}=\mathbf{2 9}(\mathbf{\%})$ & $9(10.0)$ \\
\hline RAEB-1 & $14(15.6)$ & $16(17.8)$ \\
\hline RAEB-2 & $15(16.7)$ & $\mathbf{N}=\mathbf{2 3}(\mathbf{\%})$ \\
\hline Diagnostic category $\mathbf{3}$ & $\mathbf{N}=\mathbf{2 1}(\mathbf{\%})$ & $23(25.6)$ \\
\hline s-AML & $21(23.3)$ & $\mathbf{N}=\mathbf{6}(\mathbf{\%})$ \\
\hline Diagnostic category $\mathbf{4}$ & $\mathbf{N}=\mathbf{9}(\mathbf{\%})$ & $5(5.6)$ \\
\hline CMML-1 & $4(4.4)$ & $1(1.1 \%)$ \\
\hline CMML-2 & $5(5.6)$ & $\mathbf{N}=\mathbf{2}(\mathbf{\%})$ \\
\hline Diagnostic category 5 & $\mathbf{N}=\mathbf{0}(\mathbf{\%})$ & $2(2.2)$ \\
\hline Reactive changes & - & \\
\hline
\end{tabular}

CMML: Chronic myelomonocytic leukemia; MDS: Myelodysplastic syndrome; RCMD Refractory cytopenia with multilineage dysplasia; RAEB: Refractory anemia with excess blasts; RARS-T: Refractory anemia with ring sideroblasts and thrombocytosis; s-AML: Secondary acute myeloid leukemia.

consented with use of coded laboratory data for research purposes. Ethics approval was obtained from the local Ethic Commissions.

During the routine diagnostic process, bone marrow aspirates (cytomorphology) were prospectively evaluated by two hematologists and bone marrow core biopsies (histopathology) specimens by two hematopathologists within the usual diagnostic workflow. The hematology and hematopathology routine assessments were independent. For the purpose of this study, the diagnoses obtained at the primary investigation were independently re-assessed by three recognized experts in cyto- and histomorphology of myeloid disorders (LE, VUB, YB) integrating further laboratory results and the clinical background. All investigators of the study were blinded for the previous classifications. Bone marrow samples were re-examined for dysplastic features using a pre-defined assessment form based on WHO criteria [1], as shown in Supplemental Table S1. Following this diligent reevaluation process, the conclusive diagnoses derived at the primary routine assessment were slightly modified in a small proportion of cases either for the cytomorphologic or for the histopathologic categorization ( 10 cases; $11.1 \%$ of the study cohort), whereas the vast majority of cases remained categorized as before. Thus, the focus was the real-world diagnostic scenario in a university setting that was later re-evaluated (and modified in some cases) as part of this study. Only few samples $(n=6)$ had been excluded due to insufficient material quality.

For cytomorphology, bone marrow slides were May-GrünwaldGiemsa (MGG) stained. Forty-five cases underwent iron staining (Berliner Blau) and a proportion were analyzed for myeloperoxidase $(\mathrm{n}=34)$ and non-specific esterase $(\mathrm{n}=33)$. Cytomorphology classification followed WHO, 2008; categorization of a hematopoietic lineage as dysplastic required $\geq 10 \%$ dysplastic cells [1]. For histopathology, biopsy specimens were stained in the original diagnostic workup by Hematoxilin and Eosin (H\&E), periodic-Acid Schiff (PAS), iron, and Gomori silver stain (Tissue-Tek Prisma, Automated Slide Stainer, Sakura Europe). Immunohistochemistry (BOND III, Leica Biosysterms, Nussloch, Germany) was including CD3, CD42b (Novocastra), CD20, CD34, CD68, CD117, Myeloperoxidase (Dako), CD138 (AbD Serotec), CD71 (Cell Marque). Details of the parameters assessed by both techniques are given in Supplemental Table S1. A proportion of patients $(n=39)$ was analyzed by molecular methods including PCRbased methods, Sanger sequencing, or increasingly next-generation sequencing (NGS). Cytogenetics were available in 85 patients $(94.4 \%$ of all cases). More detailed information is given in Table 1 and Table S3. Results of flow cytometry were sometimes raised, but not integrated into our study. Statistics were calculated by SPSS 24 (Statistical Package for Social Science, IBM). Correlation coefficients were calculated according to Spearman. Frequencies were compared by chi square.

\section{Results}

Cytomorphology on bone marrow aspirates revealed the following diagnoses according to WHO 2008 [1] (Table 2): refractory anemia with excess blasts (RAEB, $n=29 / 90,32.2 \%$ ), followed by s-AML $(\mathrm{n}=21,23.3 \%)$, refractory cytopenia with multilineage dysplasia (RCMD, $\mathrm{n}=15,16.7 \%)$, and suspected MDS ( $\mathrm{n}=8,8.9 \%)$. In three patients $(n=3,3.3 \%)$ MDS/MPN overlap was diagnosed. Nine patients $(\mathrm{n}=9,10.0 \%)$ were classified as CMML. Two patients were diagnosed as hypoplastic MDS ( $n=2,2.2 \%)$, two as refractory anemia with ring sideroblasts (RARS), or as refractory anemia with ring sideroblasts with thrombocytosis (RARS-T, $\mathrm{n}=1,1.1 \%$ each), and one patient had MDS with isolated 5q-deletion ( $\mathrm{n}=1,1.1 \%)$.

We grouped all cases to the following five diagnostic categories: 1) MDS without increase in blasts (RCMD, RCMD-RS, RARS, RARS-T, hypoplastic MDS, MDS with 5q deletion, MDS/MPN-overlap, suspected MDS): $\mathrm{n}=31 / 90$ (34.4\%); 2) MDS with increased blasts (RAEB-1/-2): $\mathrm{n}=29$ (32.2\%); 3) s-AML: $\mathrm{n}=21$ (23.3\%); 4) CMML (CMML-1/-2): $\mathrm{n}=9$ $(10.0 \%)$. No case was assigned to category 5 (reactive changes). 
By histopathology on bone marrow specimens, the most common diagnoses in order of decreasing frequency were (Table 2): MDS RAEB $(\mathrm{n}=25 / 90,27.8 \%)$, s-AML $(\mathrm{n}=23,25.6 \%)$, suspected MDS $(\mathrm{n}=20$, $22.2 \%)$, and MDS RCMD ( $\mathrm{n}=10,11.1 \%)$. CMML was diagnosed in six cases (6.7\%). A hypoplastic MDS was detected in two cases $(2.2 \%)$. Two cases were considered reactive (2.2\%). One patient had MDS with isolated $5 \mathrm{q}$-deletion, one showed MDS/MPN overlap (1.1\% for each). Following the categories above, we found the following frequencies by histopathology: 1) MDS without blast increase: $\mathrm{n}=34 / 90$ (37.8\%); 2) MDS with blast increase: $\mathrm{n}=25$ (27.8\%); 3) s-AML: $\mathrm{n}=23$ (25.6\%); 4) CMML: $\mathrm{n}=6$ (6.7\%); 5) reactive changes: $\mathrm{n}=2(2.2 \%)$.

To compare the classification by cytomorphology and histopathology, cases were assigned to the above five categories, primarily based on cytomorphology. The highest concordance rate was found for "s-AML" (category 3) as cytomorphologic assessment was confirmed in $20 / 21$ cases (95.2\%) by histopathology. This was followed by a concordance rate of $83.9 \%$ in the category "MDS without increase in blasts" (category 1; 31 cases classified accordingly by cytomorphology were supported by the absence of blast increase and the existence of dysplastic features by histopathology in 26 cases). For category 2 "MDS with increase in blasts" the concordance rate was $72.4 \%$ (29 cases classified accordingly by cytomorphology were corresponding in 21 cases to histopathology, as there was blast increase between 5-19\%). The correspondence rate was lowest for "CMML" (category 4; detected by cytomorphology in nine cases; matched by histopathology in five cases, $55.6 \%)$. Thus, cytomorphology and histopathology were concordant in $72 / 90$ cases $(80.0 \%)$ when the total cohort was summarized.

In $18 / 90$ patients $(20.0 \%)$, the diagnostic categories were differing by cytomorphology and histopathology: by cytomorphology, they were categorized as "MDS with increase in blasts" (category 2; $\mathrm{n}=8 / 18$ ), "MDS without increase in blasts" (category $1 ; \mathrm{n}=5$ ), followed by "CMML" (category 4; $\mathrm{n}=4$ ), and "s-AML" (category 3; $\mathrm{n}=1$ ). By histopathology, most frequent was "MDS without increase in blasts" (category 1; $\mathrm{n}=8 / 18$ ) and "MDS with increase in blasts" (category 2; $\mathrm{n}=4$ ), followed by "s-AML" (category $3 ; \mathrm{n}=3$ ), "reactive changes" (category 5; $\mathrm{n}=2$ ), and "CMML" (category $4 ; \mathrm{n}=1$ ). These 18 cases could be summarized as follows: 1 ) differing results due to difficulties in determining blast percentages: $n=6 ; 2$ ) different results due to poor marrow sample quality: $n=6 ; 3$ ) different results due to difficulties in determining monocytes/monocytic blasts by histopathology: $\mathrm{n}=4$; and 4) different results due to classification as RCMD in cytomorphology and as reactive changes in histopathology: $n=2$. More detailed information about the 18 discrepant cases between cytomorphology and histopathology are given in Figures $1 \mathrm{a}-\mathrm{c}$ and Supplemental Table S4. Supplementary diagnostics like cytogenetics and molecular analyses (if available) were considered for the conclusive diagnosis (Table S4). Focusing on MDS cases as diagnosed by cytomorphology and/or histopathology, the frequency of TP53 mutations was $n=2 / 8$ in our cohort. The respective analyses were driven by the physicians in charge and should not be considered representative due to the limited sample size. In the literature, frequencies of 8.1 to $14.0 \%$ are given for TP53 mutations in MDS $[15,16]$.

There was a good correlation between both methods for investigating dysplasia in the three hematopoietic lineages (granulopoiesis: $\chi^{2}(1)=1.176, p=0.278$; erythropoiesis: $\chi^{2}$ not applicable, as all cases were dysplastic by cytomorphology; megakaryopoiesis: $\chi^{2}(1)=1.587, \mathrm{p}=0.208$ ) (Table S2A). Correlation of cytomorphology and histopathology for overall cellularity (proportion of hematopoietic tissue to adipocytes) and for cellularity of the three hematopoietic lineages was limited

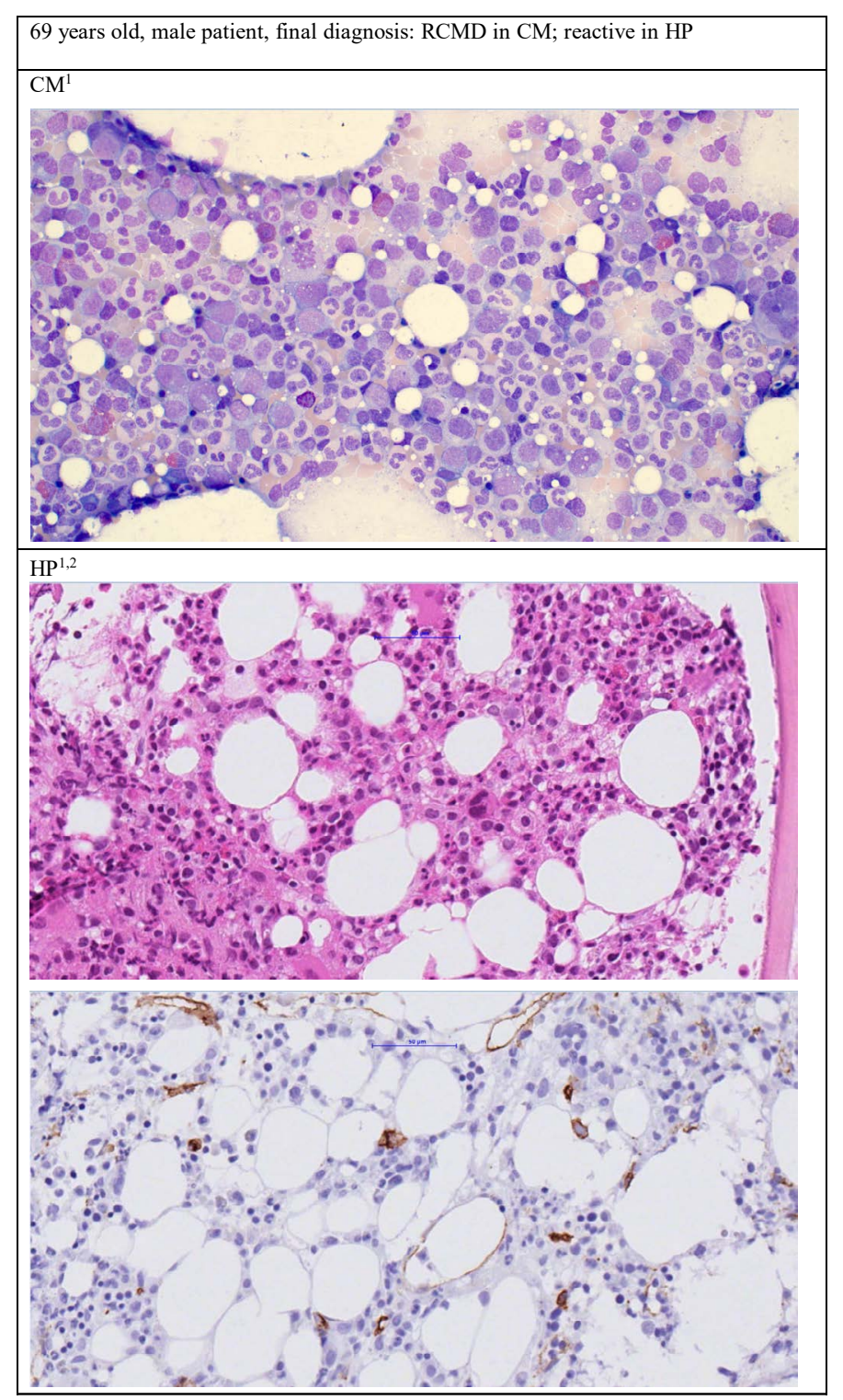

Figures 1. Examples of different diagnoses by cytomorphology and histopathology. Figure 1a. Patient's diagnosis was RCMD by cytomorphology but reactive changes by histopathology.

$1400 \times$ Zoom, Staining in CM: May-Grünwald-Giemsa, staining of first picture in HP: Hematoxillin and Eosin, ${ }^{2}$ Second picture of HP: Immunhistochemistry CD34

(overall cellularity: $\chi^{2}(4)=7.942, p=0.094$; granulopoiesis: $\chi^{2}(4)=13.252$, $\mathrm{p}=0.010$; erythropoiesis: $\chi^{2}(4)=26.764, \mathrm{p}<0.001$; megakaryopoiesis: $\left.\chi^{2}(4)=36.180, p<0.001\right)$ (Supplemental Table S2B). Cytomorphology and histopathology were concordant in the subcategorization of bone marrow blasts (thresholds $<5 \%, 5$ to $9 \%, 10$ to $19 \%$, and $\geq 20 \%$ ) in only two thirds of the 82 cases that were evaluable by both techniques for this parameter $(\mathrm{n}=54 / 82 ; 65.9 \%, r=0.69, \mathrm{p}<0.0001)$. Assignment of cells to the monocytic lineage revealed a similar lower concordance between cytomorphology and histopathology $(n=40 / 58 ; 69.0 \%, r=0.31$, $\mathrm{p}=0.0197$; only 58 cases were evaluable due to limited availability of immunohistochemistry for CD68 and due to limited sample quality).

\section{Discussion}

In this study, bone marrow samples from patients with different myeloid entities underwent a standardized re-evaluation of the initial 

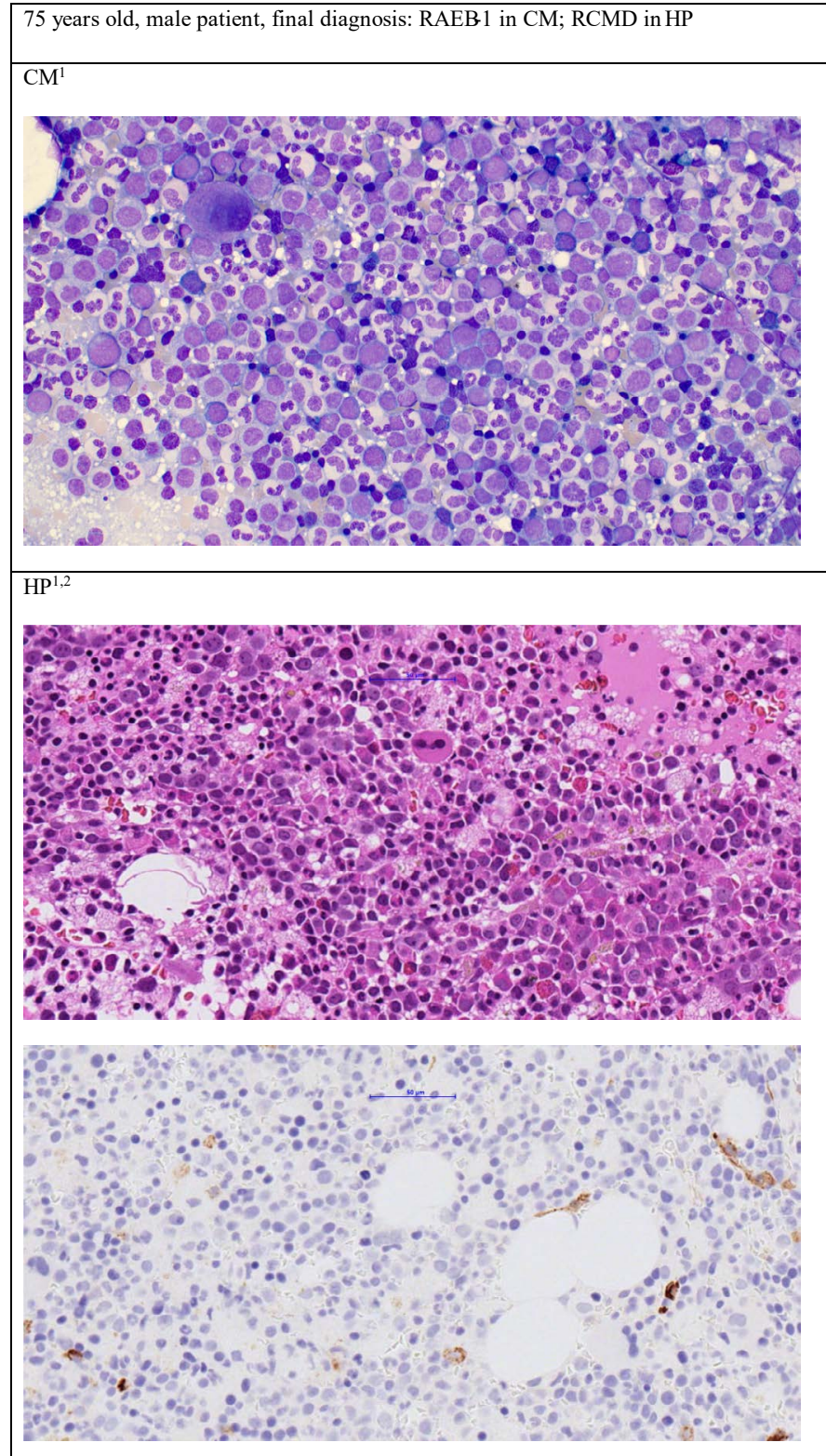

Figure 1b. Patient's diagnosis was RAEB-1 by cytomorphology but RCMD by histopathology.

${ }^{1} 400 \times$ Zoom, Staining in CM: May-Grünwald-Giemsa, staining of first picture in HP: Hematoxillin and Eosin, ${ }^{2}$ Second picture of HP: Immunhistochemistry CD34a

routine diagnostic assessment by experienced hematologists and hematopathologists. The "real-world" diagnostic classification of bone marrow samples was retrospectively re-evaluated in the context of this study. Both techniques - cytomorphology and hematopathology placed $80 \%$ of bone marrow samples into the same diagnostic category and thus showed a good correlation. Interestingly, the concordance rate was highest in "s-AML" followed by "MDS without increase in blasts", and "MDS with increase in blasts" (95\% vs. $84 \%$ vs. $72 \%$ of all cases). In contrast, the concordance rate was low for "CMML" with $56 \%$ only. This demonstrates the limitations of both phenotypic methods for some cases of myeloid malignancies and underscores their complementarity. Furthermore, this emphasizes in particular the value of molecular diagnostics for the diagnosis of CMML, with $>90 \%$ of patients carrying mutations [17]. Due to the period of study ranging
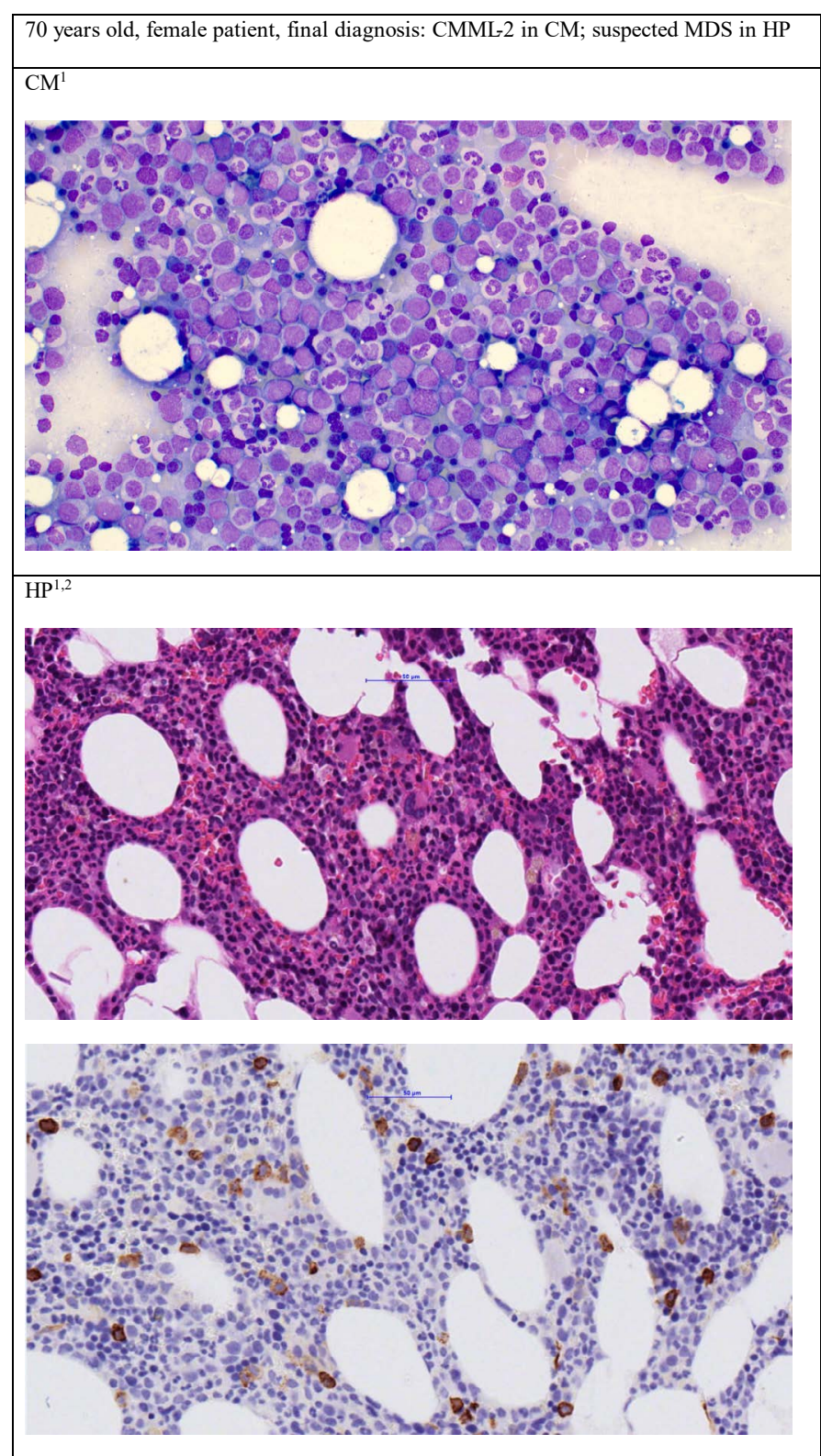

Figure 1c. Patient's diagnosis was CMML-2 by cytomorphology but suspected MDS by histopathology.

${ }^{1} 400 \times$ Zoom, Staining in CM: May-Grünwald-Giemsa, staining of first picture in HP: Hematoxillin and Eosin, ${ }^{2}$ Second picture of HP: Immunhistochemistry CD34

back as far as 2007 , only a proportion of the respective patients had been investigated by NGS.

As demonstrated in our cohort, it was possible to assign a conclusive diagnostic entity in $20 \%$ of the cases with discrepant initial evaluations based on the above-mentioned parameters. Moreover, hematology and histopathology showed limited concordance regarding the assessment of blasts, monocytic cells, and cellularity, whilst there was a high concordance rate regarding dysplasia.

These results demonstrate the limitations in correlating cytomorphology and histopathology in some cases of myeloid malignancies, even following stringent and repetitive diagnostic assessments. They emphasize the need for a comprehensive diagnostic review in a patient-for-patient setting when a myeloid malignancy is 
suspected or confirmed, especially in cases with borderline morphologic features. Finally, they suggest the value of including the clinical history (e.g. the dynamics of peripheral blood values, infections, medications, comorbidities, or other reasons for dysplasia/cytopenia [18]) and results of all relevant techniques such as cytogenetics, array comparative hybridization, FISH, and molecular genetics/NGS, to obtain the most appropriate diagnostic categorization in discrepant cases [10]. Finally, the role of flow cytometry for diagnosis and risk stratification for MDS patients is increasing [19].

Patients with myeloid malignancies with differing results of cytomorphologic and histopathologic assessment, remain a challenge for all those involved in the diagnostic and clinical aspects of patients' care. Frequently, such discrepancies will result in excessive diagnostic work-up and therapeutic insecurity. This may result in re-evaluation or even interruption of implemented therapies and repetitive discussion of the respective cases amongst physicians, and lab staff. Interdisciplinary boards are therefore essential to try to reach a final consensus on diagnosis, especially in difficult cases of suspected or myeloid malignancy with borderline morphological features. The combination of cytomorphologic and histopathologic assessment with clinical and laboratory data as well as genetic parameters are essential cornerstones in order to achieve high diagnostic accuracy in an interdisciplinary setting.

\section{Ethics approval and consent to participate}

Ethics approval was obtained from the local Ethic Commission. Bern: KEK: 339/14; PB_2016-01917, PB_2017/00699; Munich: EK 2128/08, EK 409/14 S.

\section{Consent for publication}

Not applicable.

\section{Availability of data and materials}

The primary datasets used and analyzed during the current study are available from the corresponding author on reasonable request.

\section{Competing interests}

The authors have no conflicts of interest to declare.

\section{Funding}

The study did not receive any funding.

\section{Authors' contributions}

KSG, KS, CMT, NB, ML performed research and collected clinical data; LE, YB, VUB performed the microscopic analysis; LE, JF, YB, VUB analyzed data and wrote the manuscript; TA performed the statistical analysis; VUB and YB designed the research, supervised the study, and revised the manuscript; all authors have read and approved the final manuscript.

\section{Acknowledgement}

The authors thank all coworkers in routine diagnostics for their dedicated work.

\section{References}

1. Swerdlow SH, Campo E, Harris NL, Jaffe ES, Pileri SA, et al. (2008) WHO classification of tumours of haematopoietic and lymphoid tissues. World Health Organization Classification of Tumours. (4th Edn) IARC Press: Lyon.
2. Germing U, Strupp C, Giagounidis A, Haas R, Gattermann N, et al. (2012) Evaluation of dysplasia through detailed cytomorphology in 3156 patients from the Dusseldorf Registry on myelodysplastic syndromes. Leuk Res 36: 727-734.

3. Aul C, Gattermann N, Schneider W (1992) Age-related incidence and other epidemiological aspects of myelodysplastic syndromes. Br J Haematol 82: 358-367.

4. Bonadies N, Feller A, Rovo A, Ruefer A, Blum S, et al. (2017) Trends of classification, incidence, mortality, and survival of MDS patients in Switzerland between 2001 and 2012. Cancer Epidemiol 46: 85-92.

5. Ma X (2012) Epidemiology of myelodysplastic syndromes. Am J Med 125: S2-S5.

6. Ma X, Does M, Raza A, Mayne ST (2007) Myelodysplastic syndromes: incidence and survival in the United States. Cancer 109: 1536-1542.

7. Haferlach T (2012) Molecular genetics in myelodysplastic syndromes. Leuk Res 36 1459-1462.

8. Kohlmann A, Bacher U, Schnittger S, Haferlach T (2014) Perspective on how to approach molecular diagnostics in acute myeloid leukemia and myelodysplastic syndromes in the era of next-generation sequencing. Leuk Lymphoma 55: 1725-1734.

9. Nybakken GE, Bagg A (2014) The genetic basis and expanding role of molecular analysis in the diagnosis, prognosis, and therapeutic design for myelodysplastic syndromes. J Mol Diagn 16: 145-158.

10. Bacher U, Shumilov E, Flach J, Porret N, Joncourt R, et al. (2018) Challenges in the introduction of next-generation sequencing (NGS) for diagnostics of myeloid malignancies into clinical routine use. Blood Cancer J 8: 113.

11. Kwok B, Hall JM, Witte JS, Xu Y, Reddy P, et al. (2015) MDS-associated somatic mutations and clonal hematopoiesis are common in idiopathic cytopenias of undetermined significance. Blood 126: 2355-2361.

12. Malcovati L, Cazzola M (2015) The shadowlands of MDS: idiopathic cytopenias of undetermined significance (ICUS) and clonal hematopoiesis of indeterminate potential (CHIP). Hematology Am Soc Hematol Educ Program 2015: 299-307.

13. Font P, Loscertales J, Benavente C, Bermejo A, Callejas M, et al. (2013) Inter-observe variance with the diagnosis of myelodysplastic syndromes (MDS) following the 2008 WHO classification. Ann Hematol 92: 19-24.

14. Font P, Loscertales J, Soto C, Ricard P, Novas CM, et al. (2015) Interobserver variance in myelodysplastic syndromes with less than $5 \%$ bone marrow blasts: unilineage vs. multilineage dysplasia and reproducibility of the threshold of $2 \%$ blasts. Ann Hematol 94: 565-573.

15. Kita-Sasai Y, Horiike S, Misawa S, Kaneko H, Kobayashi M, et al. (2001) International prognostic scoring system and TP53 mutations are independent prognostic indicators for patients with myelodysplastic syndrome. Br J Haematol 115: 309-312.

16. Kulasekararaj AG, Smith AE, Mian SA, Mohamedali AM, Krishnamurthy P, et al (2013) TP53 mutations in myelodysplastic syndrome are strongly correlated with aberrations of chromosome 5, and correlate with adverse prognosis. Br J Haematol 160: $660-672$.

17. Patnaik MM, Tefferi A (2016) Cytogenetic and molecular abnormalities in chronic myelomonocytic leukemia. Blood Cancer J 6: e393.

18. de Swart L, Smith A, MacKenzie M, Symeonidis A, Neukirchen J, et al. (2017) Cytomorphology review of 100 newly diagnosed lower-risk MDS patients in the European LeukemiaNet MDS (EUMDS) registry reveals a high inter-observer concordance. Ann Hematol 96: 1105-1112.

19. Duetz C, Westers TM, van de Loosdrecht AA (2019) Clinical Implication of MultiParameter Flow Cytometry in Myelodysplastic Syndromes. Pathobiology 86: 14-23.

Copyright: (C2019 Engelbrecht L. This is an open-access article distributed under the terms of the Creative Commons Attribution License, which permits unrestricted use, distribution, and reproduction in any medium, provided the original author and source are credited. 